\title{
The evidence for homeopathy
}

\begin{abstract}
Homeopathic medicine has been deemed improbable because it challenges the classical laws of physics and chemistry and no clear mechanisms of action has been identified. This paper argues that such dismissal is not scientific but belies an irrational position. The scientific character of a theory should be determined by the amount of reliable evidence available from all tiers of the evidentiary pyramid of evidence-based-medicine. This article reviews how homeopathy does have factual evidence, even if it doesn't have sufficient evidence from the highest tier to dispel all doubts of its scientific soundness.
\end{abstract}

Keywords: evidence, homeopathy, skepticism, biological effects, homeopathy, double blinded
Volume 3 Issue 5 - 2016

\author{
Bernardo A Merizalde \\ Department of Psychiatry and Human Behavior,Thomas \\ Jefferson University, USA
}

Correspondence: Bernardo A Merizalde, Department of Psychiatry and Human Behavior, Thomas Jefferson University, 90 I Walnut St, Philadelphia, PA 19107, USA, Tel +| 215-5038890,Email bermeriz@bmerizaldemd.com

Received: December 13, 2015 | Published: May 02, 2016

\section{Introduction}

Skepticism regarding homeopathy is based on the belief that there is no evidence to support its efficacy and that any effect is just a placebo because it can't work according to laws of physics and chemistry. However, when considering all of the facts, there is sufficient evidence to conclude that homeopathy does have biological effects in-vitro and in-vivo and can be a viable therapeutic modality. The question is not whether or not there is evidence, this would be an all-or-none scheme, and as such, a false proposition; the issue is what type of evidence there is and its quality; it is not about whether or not there is an ideal set of evidence, but whether there is any evidence at all; there is, as well shall review.

Prevalent conventional standards for scientific evidence comprise a hierarchy that holds meta-analysis of randomized controlled studies at the top; followed by, systematic reviews; then, randomized controlled studies; and at the lower end: observational studies, expert opinions, individual observations (case reports), and physiological experiments.

The available evidence for homeopathy has to be weighed on its own merit within acceptable scientific standards. Prominent researchers have pointed out that Complementary Alternative Medicine (CAM), which includes homeopathy, needs to be evaluated by the same standards as conventional medicine, but these are not sufficient. The additional research standards that apply must go beyond the evidence hierarchy noted above. The diversity and complexity of CAM models needs to be addressed with assumptions of their world views, as well as the politically controversial nature of such practices and the paucity of fully trained investigators needed to perform such research with integrity and expertise. Without this training and knowledge, development of proper protocols and study replication will be faulty. ${ }^{1}$

As homeopathy must follow conventional standards of research to prove its validity, while considering the additional standards required due to its unique characteristics, it is only fair that it should not be held to a higher standard than conventional medicine but be given the right of an equal ground, even if this is repugnant to the theoretical sensitivities of its detractors.

For example, homeopathy is charged for not having evidence from randomized control trials to prove its validity, yet, past reviews of commonly used conventional treatments has revealed that the average base for clinical decisions, in conventional medicine, based on Randomized Controlled Trials (RCT), was 23\% for evidence of positive effectiveness, and $21 \%$ of treatments had insufficient evidence of any effect. ${ }^{2}$ A summary of studies on the percent of practices around the world that base clinical decisions based on RCT showed that about $11 \%$ of primary care practices have such a support. ${ }^{3}$ A more recent study showed that this number, of either beneficial, or likely to be beneficial, efficacy of conventional treatments, has increased only to about $36 \%$, in the last 13 years. Half of the currently prescribed treatments lack evidence of efficacy. ${ }^{4}$ It is only fair that homeopathy be given the courtesy of an unbiased assessment of its available data.

When we look at the evidence for homeopathy according to the hierarchy of evidence we find that in fact, there is evidence from each of the hierarchical levels of evidence. From the highest tier, there are four comprehensive, independent systematic reviews, metaanalysis, that have evaluated available clinical trials in homeopathy. They have controlled for randomization, blinding, sample size, and other methodological criteria that control bias. Though the quality of clinical research in homeopathy is low, there is a surprising number that show positive results. ${ }^{5}$

\section{Meta-analysis and randomized controlled studies}

Kleijnen \& Knipschild, et al., ${ }^{6}$ evaluated 60 homeopathic trials and concluded that they would accept homeopathy's efficacy if only there was a plausible mechanism of action. ${ }^{6}$ This is not a sound conclusion, since we know, for example, that Acetominophen has no know mechanism of action, as well as a number of antibiotics, and these are part of the above mentioned conventional treatments without evidence of efficacy or mechanism of action. Should we stop using Acetominophen or certain antibiotic because there is no known mechanism of action?

It is true that the evidence from the top two sets is not compelling for homeopathy, but it is not totally absent as is contended by sceptics. The quantity of the evidence is small, derived from a few metaanalysis, and from a number of well performed randomized controlled trials, however, even if small, it should be counted as evidence and not just dismissed with prejudicial bias; that is not scientific.

It is ironic that homeopaths were among the first, to use double blinded experiments in human subjects. Those initial results, conducted by practicing homeopaths, were inconclusive. ${ }^{7,8}$

As noted above, there are other types of evidence, besides metaanalysis and RTCs. There are observational studies, case studies, and case reports which should be given their proper value, even if it is not seen as desirable according to the hierarchy of evidence-it is still 
evidence, if it proves to be qualitative reliable. Many observational studies have been found to prove equal results as RCT, as long as they are of sound methodologically quality. ${ }^{9}$

Concato \& Shah, et al., ${ }^{10}$ concluded, from their research comparing RCT, and meta-analyses of cohort or case-control studies in several clinical areas, that the observational studies did not systematically overestimate the magnitude of the treatment effects. And they stated: "the popular belief that only randomized, control trials produce trustworthy results and that all observational studies are misleading does a disservice to patient care, clinical investigation, and the education of healthcare professionals". ${ }^{10}$

In fact, the golden standard, the RCT, can also be marred by the same problems as non-randomized trials with over and underestimation of effects, inadequate concealment, and methodological flaws ${ }^{11}$. This has been found in some of the RCT trials included in meta-analysis of homeopathy, where the trials were not performed according to standard homeopathic methodology and yet were used to attempt to disprove homeopathy's efficacy. Accordingly, the current critique of evidence in homeopathy ignores the fact that evidence-based medicine principles require the informed use of all types of evidence in patient care. This involves all the evidence available from the top to the bottom of the hierarchy. ${ }^{12-14}$

In addition, the current evidence hierarchy is far too simplistic particularly when considering the complex interventions in many CAM practices. Much of the research on therapeutics are designed as a single medicine for a particular disease, with strict control of variables, and that is an ideal situation, and one to be fulfilled, if possible, but strict laboratory conditions don't usually represent real life situations. Patients suffer from complex and interactive factors, which may, or may not, be additive, and are virtually impossible to replicate, or isolate, in controlled research environments. These complex physical, psychological, and sociological factors can't be reduced to a single, concrete, measure; many times, the most important outcome for the patient is a subjective quality of life measurement. These personal experiences can only be identified through properly conducted qualitative research, which is far from what can be done with an RCT and closer to what is addressed with an observational study.

Usually, the proper choice of a homeopathic medicine, for a particular patient, for a particular condition in that specific patient (according to the unique way in which this patient manifest a particular disease), requires a thorough assessment of all, of the above mentioned, complex, factors present in the patient's clinical presentation. This unique methodological process is very hard to replicate in a RCT. This doesn't mean more concrete evidence should not be sought; laboratory and diagnostic tests should be expected to confirm diagnosis, progress, prognosis, and outcome.

\section{Real world ground for evidence}

Pluralism in the variety of evidence that can and should be gathered, in order to assure that the best evidence is included, is the wisest way to assure the acquisition of truth; sometimes, the best evidence is not causal, objective, additive, or clinical. The search for an ideal of elegant uniformity may not necessarily offer the best quality of data from a world that shows contrasts, discontinuities and complexity.

Accordingly, research domains can be arranged in a semihierarchical schema, forming multiple "rooms" within an "evidence house," each providing an important, or most important, aspect in a particular scientific investigation. One "room" may include data regarding mechanisms of action and causality; another may contain laboratory studies and basic research; another, observational studies; another randomized control trials; another qualitative case reports; another, epidemiological reports. In this way we can have greater assurance that the final data conforms to real life situations (external validity) and that interventions can be generalized to standard practices. ${ }^{1}$

Besides the highest hierarchy of evidence, the meta-analysis of randomized controlled trial, and the RCT, we find systematic reviews showing evidence of an effect from homeopathic preparations. A review of studies of agents used in warfare and using dilutions of the substances to induce tolerance showed some effect from such diluted substances. ${ }^{15}$

\section{Clinical research cohort studies}

Another comprehensive review of clinical studies conducted, including early studies published in German, concluded that homeopathy is clinically effective. In recognition of this valuable work Dean was awarded the first Hans Walz Prize of the Robert Bosch Foundation in October 2003. The aim of this prize is to promote research in the history of homeopathy outside the Robert Bosch Foundation's Institute for the History of Medicine. ${ }^{16}$

Cohort studies, another tier in the evidence hierarchy, using homeopathy, have shown that disease severity and quality of life demonstrated marked and sustained improvements following homeopathic treatment period. The researcher's findings indicate that homeopathic medical therapy may play a beneficial role in the longterm care of patients with chronic diseases. ${ }^{17}$ The use of homeopathic medicines can also decrease the cost of pharmaceutical treatment by as much as $54 \%$ in some cases. ${ }^{18}$

Another prospective cohort study showed that there was an improvement of quality of life as well as tendency offatigue symptoms to decrease in cancer patients under complementary homeopathic treatment. These results need to be replicated, ideally, with a paired controlled group. ${ }^{19}$

\section{In-vitro studies}

There are various other sets of experiments, evaluating various models, from basophile degranulation to thyroxin-dependent stimulation of metamorphosis in tadpoles; from in-vitro to in-vivo studies; which show that homoeopathically prepared substances, diluted and succussed beyond Avogadro's number are biologically active. However, these effects have either not been replicated, or the replication has shown inconsistent results. The conclusion is that an effect has been evident, but it is difficult to reproduce consistently, in the laboratory. ${ }^{20}$

In another set of experiments, Van Wijk has demonstrated in a series of studies that the similar principle can be evidenced biologically. Cell cultures were treated with low levels of toxic substances. The cell cultures expressed different patterns of stress proteins, depending of the substance used. The capacity for survival of these cells was positively correlated with the degree of similarity of the substance; the correlation was highly significant. ${ }^{21,22}$

\section{Case series and single case reviews}

Though there are yet to be published in modern journals a case series of cases, these cases could be considered cohort studies since these groups were from among the same populations, treated with homeopathy. Andre Saine, ${ }^{23}$ has collected statistics on the use of homeopathy in epidemic diseases: cholera, scarlet fever, yellow fever, and influenza pneumonia; with data from thousands of patients treated 
successfully with homeopathy and saving lives, when compared with the standard of care at the time. ${ }^{23}$

There are also hundreds of cases published in hundreds of homeopathic journals, many of them could be considered today beyond question to be valid cases that responded to homeopathic treatment, since they were not self-limiting conditions, and spontaneous remission in those questions is not probable. The collection of these cases is not easy since such publications are stored in various libraries around the world, and have not yet been digitized. The homeopathic community is currently working very hard on setting up data collection systems to prove that homeopathic medicine is, beyond doubt, clinically effective, even if no viable mechanism of action has been elicited.

A committee of researchers has developed a set of guidelines for the publication of case reports. These guidelines have been accepted by major peer-reviewed journals and will likely contribute to using reliable case reports as high quality evidence. The CARE (Case Report) guidelines provide a framework that supports transparency and accuracy in the publication of case reports, and the reporting of information from patient encounters. ${ }^{24-27}$

\section{Conclusion}

After reviewing the actual evidence available for homeopathy I conclude that there is sufficient evidence to validate homeopathy as a viable medical treatment if not provide sufficient data to justify funding for larger, well designed and controlled studies, free of assumptions and biases, that could demonstrate, conclusively, that homeopathy is an extraordinary medical treatment capable of diminishing the suffering of people in a cost effective manner. This evidence comes from the large numbers of cases, and case series through over 200years of homeopathy's existence; from cohort studies; from in-vitro studies; and from meta-analysis, even though the evidence from them is small, which is not the same as non-existent

\section{Acknowledgments}

None.

\section{Conflicts of interest}

Author declares there are no conflicts of interest.

\section{Funding}

None.

\section{References}

1. Jonas W. Building an Evidence House: Challenges and Solutions to Research in Complementary and Alternative Medicine. Forsch Komplementarmed Klass Naturheilkd. 2005;12(3):159-167.

2. Ezzo J, Bausell B, Moerman D, et al.Reviewing the Reviews: How Strong is the Evidence? How clear are the conclusions? Int $J$ Technol Assess Health Care. 2001;17(4):457-466.

3. Pelletier K. Conventional and Integrative Medicine: Evidence Based? Sorting Fact from Fiction. FACT. 2003;8(1):3-6.

4. BMJ. Clinical Evidence efficacy Categorizations. BMJ Clinical Evidence. 2014

5. Jonas W, Kaptchuk T, Linde K. A Critical Overview of Homeopathy. Ann Intern Med. 2003;138(5):393-399.

6. Kleijnen J, Knipschild P, Riet G. Clinical Trials of Homeopathy. BMJ 1991;302(6772):316-323
7. Stolberg M. Homeopathy on Trial: The First Double-Blind Experiment in Medical History in the year 1835. Muchner Medizinische Wocheschrift. 1996;138:364-366.

8. Kaptchuk T. Intentional Ignorance: A History of Blind Assessment and Placebo Controls in Medicine. Bull Hist Med. 1998;72:389-433.

9. Benson K, Hartz A. A comparison of observational studies and randomized controlled trials. $N$ Engl J Med. 2000;342(25):1878-1886.

10. Concato J, Shah N, Horowitz R. Randomized, controlled trials, observational studies and the hierarchy of research designs. $N$ Engl $J$ Med. 2000;342(5):1887-1892.

11. Kunz RA, Oxman AD. The Unpredictability Paradox: Review of Empirical Comparisons of Randomised and Non-Randomised Clinical Trials. BMJ. 1998;317(7167):1185-1190.

12. Guyatt G, Rennie D. The Evidence-Based Medicine Working Group. User's Guides to the Medical Literature: a manual for evidence-based clinical practice. American Medical Association Press, Chicago, USA. 2002.

13. Guyatt G. Evidence-Based Medicine: Past, Present, and Future. MUMJ. 2003;1(1):27-32.

14. Sackett D, Rosenberg W, Gray JA, et al.Evidence Based Medicine: What it is, What it isn't. BMJ. 1996;312(7023):71-72.

15. Szeto AL, Rollwagen F, Jonas WB. Rapid Induction of Protective Tolerance to Potential Terrorist Agents: A systematic review of low and ultra-low dose research. Homeopathy. 2004;93(4):173-178.

16. Dean ME. The Trials of Homeopathy: Origins, Structure and Development. The Journal of Alternative And Complementary Medicine. 2005;11(5):871-874.

17. Witt C, Ludtke R, Baue R, et al. Homeopathic medical practice: Longterm results of a cohort study with 3981 patients. BMC Public Health. 2005;5:115-123.

18. Frenkel M, Hermoni D. Effects of homeopathic intervention on medication consumption in atopic and allergic disorders. Altern Ther Health Med. 2002;8(1):76-79.

19. Rostock M, Naumann J, Guethlin C, et al. Classical homeopathy in the treatment of cancer patients - a prospective observational study of two independent cohorts. BMC Cancer. 2011;11:19.

20. Walach H, Jonas W, Ives J, et al.Research on Homeopathy: State of the Art. J Altern Complement Med. 2005;11(5):813-829.

21. Van Wijk R, Weigant F. Cultured Mammalian Cells in Homeopathy Research: The Similia Principle in Self-Recovery. Universiteit Utrecht, Germany. 1994.

22. Van Wijk R, Weigant F. The Similia Principle in Surviving Stress: Mammalian Cells in Homeopathy Research. Utrecht University, Germany. 1997.

23. Saine A. Homeopathy: Great Medicine or Dangerous Pseudoscience? Pure Homeopathy. 2015.pp.63-113.

24. Gagnier JJ, Kienle G, Altman DG, et al. The CARE guidelines: consensus-based clinical case report guideline development. Journal of Clinical Epidemiology. 2013;67(1):46-51.

25. Milgrom LR. Patient-Practitioner Remedy (PPR) Entanglement. Part 3: Refining the Quantum metaphor for homeopathy. Homeopathy. 2003;92(3):152-160.

26. Torres J. On the Physical Basis of succussion. Homeopathy. 2002;91(4):221-224.

27. Walach H. Entanglement Model of Homeopathy as an example of generalized entanglement predicted by weak quantum theory. Forsch Komplementarmed Klass Naturheilkd. 2003;10(4):192-200. 